\title{
Organisations et développement durable : analyse comparée des discours d'acteurs locaux
}

Gaël Plumecocq

\section{(2) OpenEdition \\ 12 Journals}

Édition électronique

URL : http://journals.openedition.org/ei/148

DOI : $10.4000 /$ ei. 148

ISSN : 2553-1891

Éditeur

Association Économie et Institutions

Édition imprimée

Date de publication : 15 octobre 2010

Pagination : $31-57$

ISSN : 1775-2329

\section{Référence électronique}

Gaël Plumecocq, «Organisations et développement durable : analyse comparée des discours d'acteurs locaux ", Économie et institutions [En ligne], 15 | 2010, mis en ligne le 31 janvier 2013, consulté le 07 mai 2019. URL : http://journals.openedition.org/ei/148 ; DOI : 10.4000/ei.148 


\section{Organisations et développement durable : analyse comparée des discours d'acteurs locaux ${ }^{1}$}

\section{Gaë1 Plumecocq ${ }^{2}$}

Depuis une quarantaine d'années les crises économiques se succèdent, jetant un sérieux doute sur la capacité du système marchand à produire bien-être et développement. Deux critiques semblent particulièrement se dégager : d'une part, l'idée que le capitalisme serait un système non durable ou non soutenable (Harribey, 1998) ; et d'autre part, sur le rôle plus spécifique des entreprises dont la vision en termes de création de valeur apparaît trop étroite (Gendron, 2000). Il existe sans doute des liens forts entre ces deux critiques, la dynamique du développement durable (DD) prescrivant, en particulier, l'adoption d'une nouvelle éthique de la production. Celle-ci, comprise comme l'ensemble des devoirs incombant à une entreprise (qualité sanitaire des produits, conditions de sécurité des employés, etc.), conduirait notamment à une meilleure prise en compte de l'impact de leurs activités sur l'environnement naturel. À cet égard, la problématique de la Responsabilité Sociale et Environnementale des entreprises (RSE), bien que plus ancienne, semble reconfigurée par la mobilisation de justifications invoquant une participation au DD. Pour autant, il existe sans doute plusieurs manières d'articuler DD et RSE. Certains voudraient, par exemple voir dans les entreprises, un acteur clé de la dynamique de $\mathrm{DD}$. Le Groupe de Lisbonne rappelle ainsi que l'entreprise joue un rôle central dans la mise en place des moyens techniques d'un DD dans la mesure où elle " est la seule organisation à avoir su se transformer en un joueur "mondial" [... et où] elle possède un réel pouvoir de décision " (cité par Zaccaï, 2002, p.291). D'autres, en invoquant les évolutions managériales des entreprises le passage d'une logique pyramidale à une gestion procédurale qui contribuent à effacer ses frontières (Stéphany, 2003), réintroduisent l'entreprise dans l'organisation globale du développement économique. Il s'agirait alors d'appliquer dans l'entreprise des normes décidées à l'extérieur de ses murs.

\footnotetext{
1 Je tiens à remercier Philippe Abecassis pour son assistance matérielle dans le traitement des données notamment effectué sur le logiciel Alceste. Toutes interprétations abusives ou maladresses dans les manipulations paramétriques, relevant évidemment de ma seule responsabilité. Merci également aux relecteurs pour leurs commentaires sur ce texte.

2 Doctorant, EconomiX, UMR 7166, Université Paris Ouest - Nanterre - La Défense. ATER, Université de Limoges, gael.plumecocq@u-paris10.fr, Adresse postale : 7 rue des Rossignols 87170 ISLE.
} 
L'hypothèse que nous tenterons de défendre dans ce papier, est que le mouvement de la RSE constitue une tentative de détournement des critiques issues du DD, notamment en matière de défense de l'environnement. L'adoption d'une stratégie de RSE, au nom d'une participation au DD, se fait alors davantage dans le but de défendre les intérêts des entreprises, qui tentent d'apporter une réponse crédible à la " nécessaire quête de légitimité " de ses produits (Champion et Gendron, 2005). De fait, elle contribue à freiner la dynamique de $\mathrm{DD}$, en complexifiant le système de valeurs légitimes qui sous-tend les normes molles de RSE. Cela nécessite de penser un cadre théorique, en l'occurrence celui des Économies de la Grandeur (Boltanski et Thévenot, 1991), dans lequel les pratiques, les normes et leur légitimité, se trouvent articulées de manière cohérente (partie 1). La méthode adoptée consistera alors à étudier les discours des entreprises à l'aide de la méthode Alceste, ce qui nous permettra d'établir une typologie des acteurs étudiés en fonction de leur façon de mobiliser les référentiels normatifs de RSE (partie 2). En appliquant la grille de lecture théorique présentée, nous poursuivrons en reconnaissant le rôle stratégique de la maîtrise des questions de la RSE pour les entreprises, notamment dans la promotion de leur image au regard de l'opinion publique. Cet enjeu trouve sa traduction dans le mode de légitimité qui transparaît dans la manière dont les entreprises s'approprient la problématique environnementale du DD et rendent compte de leur politique de RSE. La comparaison avec les discours des collectivités territoriales, qui mobilisent également des justifications en termes de DD, mais dont la raison d'être est, en principe, de prendre en charge la responsabilité de l'intérêt collectif ${ }^{3}$, permet de mettre en évidence l'instrumentalisation des discours de RSE par les entreprises. Le but recherché par les entreprises, est de maintenir à distance le regard de l'ensemble de la société sur ses choix stratégiques (partie 3).

\section{DD et RSE : une légitimité par les pratiques}

\subsection{Perspectives historiques}

Nous avons rappelé en introduction l'ambiguïté du lien entre DD et RSE. Historiquement pourtant, ces deux mouvements connaissent une genèse différente bien que convergente. Pour Aggeri

3 Les entreprises participent aussi de l'intérêt général, mais à la différence des organisations publiques, elles ont pour seule responsabilité d'assurer les conditions de sa reproduction organisationnelle. L'intérêt collectif est alors incidemment défendu par la recherche de la satisfaction de l'intérêt privé, comme dans le principe de la "main invisible " d'Adam Smith, au contraire des collectivités qui oeuvrent dans l'intérêt de leurs populations. 
et Godard (2006), le mouvement de la RSE accompagne les évolutions économiques du début du siècle, en particulier aux EtatsUnis, le passage à une économie financiarisée. Dans ce nouveau modèle entreprenarial, qui voit la déconnexion entre propriétaire et gestionnaire, ressurgit la question de l'éthique religieuse et de la responsabilité des dirigeants à l'égard de la société. Arrivé tardivement en Europe, le phénomène de la RSE commence à prendre de l'ampleur entre 1987 et 1992, par la rencontre, d'une part d'un mouvement poussé par les fonds de pension et le recours de plus en plus important à l'épargne salariale, motivé par une nouvelle éthique financière appelée "l'investissement socialement responsable "; et d'autre part d'une montée en puissance des revendications de type écologiste, notamment portées sur le devant de la scène médiatique par certains scandales d'ampleur internationale - par exemple, Exxon Valdez ou Tchernobyl (Stéphany, 2003). Deux caractéristiques de la RSE ressortent principalement de ces évolutions historiques: il s'agit, avant tout, d'une démarche volontaire; cette démarche vise la prise en compte des intérêts, parfois divergents, d'un ensemble étendu de ses parties prenantes. Ainsi, "la plupart des définitions de la responsabilité sociale des entreprises décrivent ce concept comme l'intégration volontaire des préoccupations sociales et écologiques des entreprises à leurs activités commerciales et leurs relations avec leurs parties prenantes. Ettre socialement responsable signifie non seulement satisfaire pleinement aux obligations juridiques applicables, mais aussi aller au-delà et investir "davantage" dans le capital humain, l'environnement et les relations avec les parties prenantes " (UE, 2001, p.7).

La genèse du DD est plus récente. Godard (1994) souligne ainsi que c'est une notion qui prend racine dans celle, plus radicale, d'écodéveloppement. Cette dernière est avancée pour la première fois lors de la Conférence des Nations Unies sur l'Environnement Humain (CNUEH) qui s'est tenue en 1972 à Stockholm. Mais porteuse d'un programme jugé trop radical, l'écodéveloppement a été remplacé par la notion plus consensuelle de DD. Si le terme apparait pour la première fois en 1980 dans un rapport de l'International Union for Conservation of Nature and Natural Resources (1980), sa popularité est largement due au succès du rapport Brundtland, commandé par les Nations Unies. Il définit le DD comme "un développement qui répond aux besoins du présent sans compromettre la capacité des générations futures de répondre aux leurs" (Brundtland, 1987, p.51).

Divers acteurs ont participé à l'" hybridation ", pour reprendre le terme d'Aggeri et Godard (2006, p.14), entre DD et RSE. Après 1992, le mouvement de refonte de l'éthique entreprenariale se solidifie dans les principes onusiens du DD, notamment à travers le 
rôle que voudraient leur faire jouer les instances internationales (ONU, OCDE), mais également en France, les pouvoirs publics locaux. Parallèlement, au cours des années 1990, des consultants britanniques diffusent un discours mettant en évidence l'intérêt stratégique d'adopter les principes du DD. Ainsi, le tournant du millénaire a-t-il vu la multiplication, et dans certains cas la remise à jour, de normes (compris comme des principes d'actions) en matière de RSE : en 1997, le Global Reporting Initiative développe des directives mondiales de DD ; en 1999, les Nations Unies mettent en place le Global Compact, instaurant un cadre de référence mondial pour la RSE ; en 2000, révision des Principes directeurs de l'OCDE, ainsi que de la Déclaration Tripartite sur les entreprises multinationales et la politique sociale (Organisation Internationale du Travail); en 2001, la France adopte une loi sur les Nouvelles Régulations Économiques obligeant les entreprises cotées en Bourse à produire un rapport sur les conséquences environnementales de leurs activités.

\subsection{Normativité et légitimité du DD.}

Sans prétendre analyser les forces à l'œuvre dans cette rencontre, nous voulons simplement insister sur le fait que, à notre sens, RSE et DD ne se positionnent pas au même niveau de normativité et donc de légitimité. La RSE constitue un cadre dans lequel des valeurs restent à définir au cas par cas en fonction de l'étendue de la responsabilité retenue. Le DD se joue, lui, à un niveau plus général. De manière plus imagée, la RSE constituerai le contenant, le DD le contenu. En ce sens, l'aspect normatif de la RSE apparait pleinement dans les principes de DD.

Après avoir étudié les textes de référence internationaux du DD, Zaccaï (2002) met en évidence cinq principes unificateurs de ce que représente un DD. 1/. Agir dans le sens d'un développement plus durable, c'est lutter pour "la promotion et la protection de l'environnement ". 2/. De ce point de vue, cela nécessite de se placer au niveau d'observation le plus pertinent, en adoptant "une vision mondiale ". 3/. Cela nécessite également de prendre en compte une temporalité propre à observer les effets produits sur l'environnement, et par conséquent à considérer "l'équilibre entre le présent et le futur ". 4/. Agir au nom du DD, c'est également rechercher "l'intégration entre les différentes composantes du développement ". $5 /$. Enfin, on remarque que la majeure partie des démarches met en avant "l'affirmation de la nouveauté du projet de DD ".

Ainsi, le DD apparaît comme un ensemble de principes normatifs plutôt que comme un cadre d'action bien balisé. De fait, cela donne lieu à de nombreuses interprétations, ainsi qu'à une grande diversité de pratiques se réclamant du DD. Cela pose alors la 
question de la manière dont les individus (ou les entreprises) se saisissent des normes, comprises comme des injonctions à agir dans un sens déterminé. Le cadre des Économies de la Grandeur, avancé par Boltanski et Thévenot (1991) permet de comprendre comment des individus peuvent appliquer des règles ou des normes qui contraignent éventuellement leur intérêt. Cela nécessite, d'une part, de supposer que les agents ont la capacité de se représenter la situation objective (objets, dispositifs d'encadrement ou de régulation, institutions...) dans laquelle la norme doit être appliquée. D'autre part, il faut supposer que ces agents disposent d'une capacité de jugement sur la légitimité de cette règle. Ainsi, dans une situation marchande, encadrée par des dispositifs qualifiant cette situation comme telle, une entreprise peut avoir intérêt à développer des procès de production plus coûteux mais moins polluants, de manière à susciter de la sympathie pour ses produits. Elle peut également chercher à préserver l'environnement par idéal écologique, ou parce que celui-ci constitue un élément reconnu du patrimoine local, national, mondial... Potentiellement, le DD constitue donc une modalité de justification des activités productives de l'entreprises, de manière à réduire l'incertitude marchande (Coutrot, 1998) en vertu de laquelle il n'y a aucune raison a priori qu'une production rencontre une demande. Ces justifications sont jugées recevables (légitimes) lorsqu'elles sont sous-tendues par une grandeur morale à l'aune de laquelle la justice est administrée, et lorsqu'elles sont mobilisées avec justesse au regard des objets sur lesquels elles prennent appui dans l'administration de la preuve - ce qui suppose que des objets soient qualifiés au regard d'une grandeur (objets qualifiés et grandeur constituent alors un "monde commun "). Ces grandeurs morales, ou principes supérieurs communs, tirent leur légitimité de grands ouvrages de philosophies politiques. Boltanski et Thévenot repèrent ainsi six ordres de valeurs légitimes. Dans chacun d'eux, il est possible de qualifier des modalités d'acceptabilité sociales des produits, mais également de traitement de la nature au sens large (Godard, 1990) :

- le monde marchand se dessine à travers l'œuvre d'Adam Smith et repose sur le principe supérieur du prix comme signe de légitimité. En tant que valeur, le prix représente le signe d'une acceptabilité sociale formulée dans une demande marchande. La nature légitime dans le monde marchand est traitée comme un produit à vendre. C'est une nature qui fait donc l'objet d'une transaction marchande (par exemple, un site qui dispose d'un droit d'entrée) et à laquelle on attache une valeur révélée dans une disposition à payer.

- le monde industriel est rattaché à l'œuvre de Saint-Simon. Le principe de justice qui ordonne les personnes est celui de l'efficacité. La nature légitime est mise en valeur via une exploitation 
productive et efficace. Le produit, en tant que résultat d'un processus combinatoire de facteurs coûteux, est donc jugé comme légitime au regard de la faiblesse de son coût de production. Plus celui-ci est faible, plus les méthodes de productions sont jugées comme efficaces et donc justes de ce point de vue, ce qui se répercute au niveau du prix de vente ${ }^{4}$.

- le monde domestique est fondé en légitimé dans l'œuvre de Bossuet, dont le principe supérieur est l'honnêteté en tant que connaissance de soi et reconnaissance de sa place dans une organisation sociale mettant en jeu le registre de la familiarité (intimité, proximité, localité, etc.). La nature domestique est donc celle avec laquelle on expérimente une relation d'intimité (l'animal domestique, le jardinet...) ou de proximité. Le produit peut également être jugé par le consommateur à l'aune de ce critère et reconnu comme légitime dans sa dimension domestique d'authenticité et de proximité (comme produit du terroir, par exemple).

- le monde civique tire sa légitimité de l'œuvre de Rousseau à travers le principe supérieur de l'égalité ou de la capacité individuelle à se dévouer pour le bien commun. La nature légitime est celle qui, par opposition au monde marchand, est gratuite et ne repose sur aucune discrimination. Les biens et services civiques sont ceux qui servent le bien commun, qui rendent un service collectif et dont l'archétype est le service public.

- le monde inspiré vient de l'œuvre de Saint Augustin et se fonde sur le principe supérieur de la grâce. La nature inspirée est celle qui invite à la méditation (quelle que soit sa forme) notamment en mettant en jeu des éléments propres au registre de l'esthétique ou de la spiritualité. Dans sa dimension entreprenariale, des éléments d'inspiration apparaissent dans les modalités de présentation du produit au public (communication, marketing, publicité, etc.).

- enfin, le monde du renom ou de l'opinion est mis en évidence à travers l'œuvre de Hobbes. Il repose sur le principe d'honneur, dont la célébrité est vue comme un signe. La nature légitime est donc, ici, celle qui est particulièrement apte (par sa position géographique, ses caractéristiques propres, sa faune ou sa flore, etc.) à mobiliser l'opinion publique. De même, le produit légitime dans le monde du renom est celui sur lequel la firme réussit à attirer l'attention du consommateur 5 .

4 Dans cette optique, la problématique du commerce équitable peut être analysée comme une tension entre deux modes de légitimité : un mode marchand et un mode industriel. Cette fracture se révèle dans écart d'évaluation en rapport avec ces deux principes: le prix du produit (évaluation marchande) et son coût de production (évaluation industrielle).

5 Pour une présentation d'exemples de la manière dont le phénomène de la RSE recombine des éléments d'inspiration et d'opinion pour faire vendre, on pourra se référer à Laville (2002). 
Ces figures de justification sont évidemment des formes pures. Dans la réalité sociale, les arguments mis en jeux ou les procédures d'objectivation des mondes communs se constituent souvent d'éléments qualifiables dans plusieurs mondes. Le mode de légitimité peut alors prendre plusieurs formes. En particulier celle du compromis entre deux grandeurs morales. Ce dernier est de fait moins stable et durable que les modalités pures. Il semble toutefois que les débats qui se constituent autour des questions d'environnement naturel répondent à cette figure particulière de justification (Godard, 1990 et 2004 ; Thévenot et al., 2000). C'est au regard de cette mixité de principes, que nous tenterons de qualifier les modalités de reconfigurations des enjeux de légitimité produites par la problématique du DD, mais également les risques qu'elles induisent dans la démarche globale. Pour ce faire, on étudiera les discours d'entreprises adoptant ostensiblement des démarches responsables. Il s'agira, ensuite, de traduire les classes de vocabulaires dégagées par Alceste, en termes d'enjeux de légitimité.

\section{Ce que disent les entreprises de la RSE}

Ces ordres de grandeur transparaissent dans les discours d'acteurs. Dans une première analyse, nous avons interrogé neuf acteurs porteurs de la RSE : cinq entreprises (No, Ca, Ok, Mt, Bo), trois associations $(\mathrm{EP}, \text { le } \mathrm{CE}, \mathrm{AL})^{6}$ et la branche régionale d'un grand syndicat national (C) Les résultats de la classification fournis par l'analyse et présentés dans la figure 1 sous forme de classification sémantique, donnent une partition du corpus en trois classes de discours identifiées comme relevant quasi-spécifiquement d'un type particulier d'acteurs.

6 No et Ca sont deux entreprises d'installation et maintenance de réseaux informatiques pour entreprises (la première est locale, la seconde multinationale), Ok est une entreprise de prêt-à-porter pour enfants, Mt est une entreprise locale spécialisée dans l'aménagement d'espaces verts et les revêtements routiers écologiques et Bo est une entreprise industrielle de produits agro-alimentaires. Du côté associatif, EP est une association de grandes entreprises nationales pour la promotion du DD, CE est un groupement d'intérêt public local et AL est une association d'acteurs du DD. 
Figure 1 - Les classes de discours de la RSE

\begin{tabular}{|c|c|c|c|c|c|}
\hline & & \multicolumn{2}{|c|}{854 u.c.e. classées } & & \\
\hline \multicolumn{2}{|c|}{ Classe $1: 61,24 \%$} & \multicolumn{2}{|c|}{ Casse $3: 14,17 \%$} & \multicolumn{2}{|c|}{$\begin{array}{c}\text { Classe } 2: 24,59 \% \\
\text { Formes }\end{array}$} \\
\hline action & 32,93 & accord+ & 154,64 & enf +ant & 64,37 \\
\hline entreprise 23,31 & 23,31 & syndica+l & 141,81 & monde+ & 45,47 \\
\hline envoironnemental+ & 19,49 & organisat+ion & 132,34 & roubaix+ & 37,32 \\
\hline sur & 15,64 & signe+ & 84,91 & fondation+ & 37,32 \\
\hline grand & 14,66 & multinationale+ & 66,28 & an+ & 35,02 \\
\hline demarche & 14,15 & cadre+ & 59,96 & societe+ & 34,58 \\
\hline sens & 9,83 & internationale+ & 53,96 & creter & 34,51 \\
\hline motivat+ion & 9,66 & negociat+ion & 53,96 & vivre. & 32,65 \\
\hline aspect & 9,63 & contenu+ & 41,76 & burt & 32,65 \\
\hline impact+ & 9,63 & internationa+l & 37,03 & capitalis+me & 31,03 \\
\hline durable & 9,06 & representant+ & 36,60 & confi+ant & 31,03 \\
\hline plan+ & 8,47 & nation+ & 36,60 & creche+ & 29,53 \\
\hline œuvre & 8,35 & orientation+ & 36,60 & marque+ & 29,53 \\
\hline processus & 7,70 & intervention+ & 35,71 & lien+ & 28,23 \\
\hline environnement & 7,55 & force+ & 31,42 & okaid+ & 27,89 \\
\hline mise & 7,30 & contrat+ & 30,26 & magasin+ & 24,77 \\
\hline vision & 7,26 & representat+ion & 29,71 & metier+ & 22,65 \\
\hline repondre & 7,14 & prenant+ & 29,40 & cherch+er & 22,37 \\
\hline site & 7,05 & comité+ & 27,58 & cahier & 21,64 \\
\hline industri< & 6,62 & signataires & 24,35 & femme+ & 19,68 \\
\hline $\log +16$ & 6,40 & convention+ & 24,35 & argent+ & 18,53 \\
\hline securite & 6,40 & gouvernement< & 23,77 & peur+ & 18,53 \\
\hline guide & 6,40 & modele+ & 22,42 & humanis+me & 18,53 \\
\hline aujourd & 6,40 & europeen+ & 21,62 & rachet+er & 18,53 \\
\hline hui & 6,40 & relation+ & 20,61 & civil+ & 15,42 \\
\hline image & 5,99 & element+ & 20,61 & discours & 15,42 \\
\hline
\end{tabular}

- Une classe de vocabulaire (classe 1) porté par les associations et les entreprises dont les activités sont lieees à l'environnement ou à la RSE. Elle recouvre principalement trois champs: un discours technique sur l'entreprise ou l'association, ses fonctions, son organisation, ses partenaires; un discours sur la manière dont le problème du DD ou de la RSE est appréhendé, sans toutefois relever de manière significative de la RSE (ni le mot $R S E^{7}$, ni le mot responsabilité n'apparaissent comme spécifiques à cette classe) ; enfin, un discours qui met en relation les deux précédents, par la manière dont le phénomène de la RSE est internalisé dans les entreprises.

- La seconde catégorie (classe 2), représentative du discours des entreprises dont l'activité est relativement éloignée de la problématique de l'environnement ou de la RSE, recouvre également trois champs spécifiques: un discours largement autocentré sur

7 Les mots en italique font référence à des entrées du corpus apparaissant dans les classes de vocabulaire spécifique, identifiées par Alceste. 
l'entreprise (ses clients, sa fonction) mettant en jeu des éléments circonstanciés (notamment géographiques); un discours de portée très générale sur l'organisation du système social; et un discours catastrophiste de prise de conscience, tourné vers l'action. Notons que le discours de Bo, qui met en jeu des éléments communs à ces deux types de discours, apparaît comme spécifique aux deux premières classes.

- Le dernier ensemble (classe 3) reprend exclusivement le discours des organisations syndicales. Celui-ci présente un acteur tendu entre le consensus et l'affrontement, qui donne à voir une vision plus large des parties prenantes que les deux types de discours précédents. Apparaissent notamment les interrelations de l'entreprise avec son environnement géographique, à la fois proche et lointain. Le vocabulaire utilisé indique une certaine projection vers l'avenir8.

Nous avons, par ailleurs, effectué un codage particulier sur le nom de la structure d'appartenance de la personne interrogée. Les résultats obtenus permettent de repérer la spécificité relative des discours par acteurs, et d'établir une classification selon deux critères : d'abord selon le recours au registre de la globalité ; ensuite à travers les modalités de positionnement de l'entreprise par rapport aux normes de DD.

8 Nous sommes conscients de la faible validité générale du terrain d'étude au regard de la dynamique d'ensemble de la RSE ou du DD. Nous essaierons donc, dans la mesure du possible de comparer les résultats obtenus à la lumière d'exemples plus représentatifs. 
Encadré : Note sur la méthode Alceste

Comme de nombreux logiciels, Alceste permet l'identification de mondes lexicaux. Méthodologiquement, Alceste découpe le texte à analyser en segments de tailles similaires (en termes de nombre de formes, mais en tenant compte de la ponctuation). Ces segments, appelés Unités de Contexte Élémentaires (UCE), sont ensuite croisés dans un tableau à double entrée avec les mots pleins qu'ils contiennent. L'ensemble des mots pleins est défini comme le complémentaire des mots outils (conjonctions, pronoms, adverbes, prépositions etc.). Le logiciel procède alors, par itérations, à des regroupements successifs d'UCE. Il calcule pour chacun des regroupements la spécificité du vocabulaire contenu, à l'aide d'un test du khi-deux, dont la valeur n'est pas significative en soi. On estime qu'un khi-deux égal à 3,41 correspond à une probabilité de $0,1 \%$ de chance d'avoir une association fortuite entre un mot plein et un regroupement d'UCE. Cette opération est répétée une seconde fois avec une longueur d'UCE différente. La classification retenue, dite " classification hiérarchique descendante ", est, non seulement celle qui fait apparaître les regroupements dont le khi-deux est le plus élevé, mais également celle qui est la plus stable (seuls les points communs entre les deux classifications sont retenus).

Plusieurs outils complémentaires permettent une analyse plus fine du corpus et des mondes lexicaux. Il est, en effet, possible de coder le texte en effectuant une segmentation en fonction de variables définies a priori. Ici, la variable retenue concernait le type d'acteur interrogé : entreprise, association, syndicat. Nous avons également utilisé une analyse factorielle qui permet de représenter l'ensemble des formes classées (mots pleins, mots outils et variables), dans un graphique comportant un axe de moins que de nombre de classes sémantiques.

\subsection{Le registre de la globalité.}

La figure 2 représente la projection sur l'axe 1 de la "distance" de spécificité entre les discours d'acteurs (analyse factorielle). Elle est surprenant à double titre. D'abord dans la mesure où le schéma représente de fortes disparités relatives entre les différentes entreprises (Ok d'un côté, et $\mathrm{Ca}$, Mt, Bo de l'autre, avec No en position intermédiaire) qui se retrouvent disposées le long de l'axe graphique. Elle surprend, ensuite, par la proximité relative d'un certain type de discours patrona $1^{9}$ et du discours syndical.

9 L'acteur interrogé chez Ok est en effet le patron de cette entreprise. Encore une fois, insistons sur les limites des généralisations présentées ici : le discours analysé ne saurait être représentatif de celui de l'ensemble des patrons. 
Figure 2 - Axe 1 de l'analyse factorielle : considérer l'environnement global de l'entreprise.

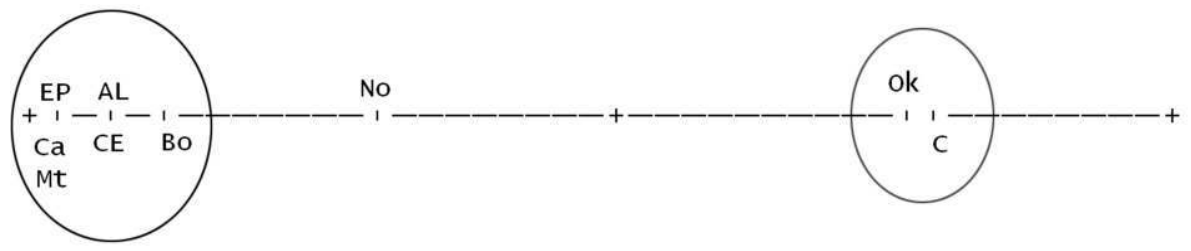

On constate que, chez $\mathrm{Ok}$, le discours en reste à un niveau de généralité assez vague, à la fois en ce qui concerne l'environnement de l'entreprise (monde, société, sociétal, communauté, collectivité), mais également sur l'organisation globale du système (capitalisme, libéralisme). Cela permet in fine de reporter la responsabilité de l'entreprise sur celle de l'organisation du système économique global. On exporte ainsi la responsabilité hors de l'entreprise, à travers une critique civique (Boltanski et Thévenot, 1991) de l'organisation capitaliste marchande, reposant sur des termes tout aussi vagues que les précédents (égalitarisme, humanitarisme) dans un discours normatif et politique du rôle de l'entreprise. Ce recours au registre de la globalité, qui fait écho au principe 3/. "du point de vue mondial " mis en évidence par Zaccaï, est également mobilisé dans d'autres contextes nationaux, notamment au Québec (Gendron, 2002). Dans ce cas précis, toutefois, les discours portent essentiellement sur des enjeux environnementaux (principalement l'eau et l'air).

Quoi qu'il en soit, le discours d'Ok entretien une certaine proximité avec celui de la C. Chacun d'eux met, en effet, l'accent sur le lien avec l'environnement global dans lequel s'insère l'entreprise (multinational, européen, pays, gouvernement, état, nation). Ce principe de globalité est donc reconnu comme une dimension importante de la dynamique de DD dans l'entreprise. On notera toutefois que chacun des discours reste spécifique, dans la forme, au type d'acteurs qui le mobilise. Ok fait référence à des modalités d'organisation économique capitalistes, tandis que $\mathrm{C}$ insiste sur les instances de régulation. Le discours syndical contribue donc à remettre l'entreprise en face de ses responsabilités, comme acteur de pouvoir, face aux institutions régulatrices qu'elles soient gouvernementales, internationales ou associatives. En cela, il apparaît comme une partie prenante centrale dans la mise en place d'une stratégie crédible de DD.

Pour autant, ces discours ne parviennent pas à mettre en évidence l'établissement de lien formalisé entre ce qu'il se passe dans, et hors de l'entreprise. Cela transparaît dans la mobilisation d'un discours très autocentré sur l'activité de l'entreprise (textile, 
achat, importer, marque, magasin), sur des éléments de proximité géographique (Roubaix, Lille), ou sur la cible de consommation (famille, enfant, ans, père). La même déconnexion se retrouve dans le discours de $\mathrm{C}$ à travers le recours fréquent à l'organisation ou l'organisme syndical(e), ou encore le syndicat, comme sujet d'action. À travers également son rôle (représentant, représentation) et son action (négocier, rassembler, revendicatif, rapport de force) dont on pourra noter qu'ils ne sont en rien spécifiques à la RSE. Pas plus d'ailleurs que le discours d'Ok. Par ailleurs, cette fracture entre le local/particulier et le global/général se retrouve chez Ok dans la manière d'utiliser les marqueurs de la personne. Cela passe par un recours massif à la première personne (je, ma, me, mes, moi, mon, nous) en opposition à eux, ils, son. Pour C, l'absence de marqueur de la personne relève de la même logique. On a recours à une dénomination propre au discours syndical où le moi est repris dans l'organisation syndicale, et le eux, dans le gouvernement, qu'il soit national ou européen.

\subsection{La référence à la norme : l'articulation du local et du global.}

De manière assez générale, la problématique de la RSE est assez étroitement liée à celle de la normalisation (Boidin et al., 2007 ; Capron, 2003). Comme pour la figure 2, la figure 3 établit la répartition des acteurs interrogés en fonction de la spécificité des discours qu'ils mobilisent. Elle met en évidence que ceux avancés par les entreprises sont diamétralement opposés à ceux des organisations syndicales. Cela ne signifie pas nécessairement une opposition de discours, mais peut représenter une complémentarité en vue de faire progresser la responsabilité de l'entreprise, chacun de ces acteurs utilisant pour décrire cette dynamique et les enjeux qui en découlent, pour l'organisation à laquelle il appartient ou pour l'entreprise, un vocabulaire exclusif. Les acteurs qui apparaissent au centre du schéma correspondent aux associations qui agissent pour la promotion du DD dans les firmes (EP, CE, AL) et des entreprises dont l'activité est directement liée à la promotion de la RSE, par le biais d'une branche d'activité spécifiquement dédiée $(\mathrm{Ca})$ ou parce que son cœur de métier est lié à l'environnement (Mt). 
Figure 3 - Axe 2 de l'analyse factorielle: discours et nature des acteurs.

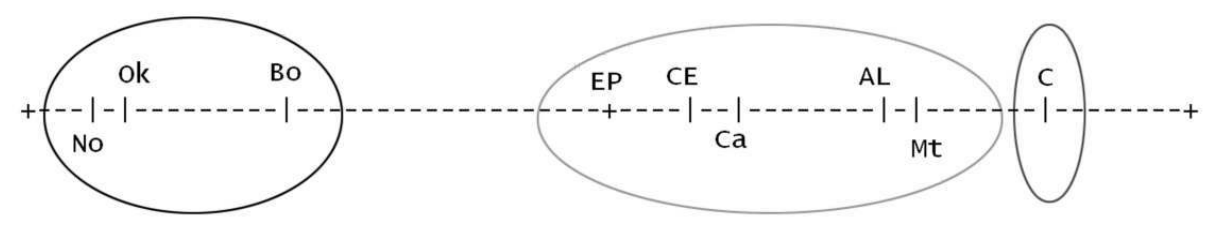

Des trois types de discours vus précédemment, chacun entretient une certaine distance relative dans leur relation à la norme. D'après ce schéma, on distingue deux cas extrêmes : celui des discours entreprenariaux, et celui des discours associatifs et syndicaux.

\subsubsection{Discours d'entreprises : l'utilité de la solidarité.}

Côté entreprise, on insiste particulièrement sur les effets dévastateurs des dégradations environnementales, sans toutefois faire référence au rôle des entreprises dans cette dynamique (si ce n'est à travers la production de déchets). Le discours est éminemment catastrophiste (mort, mourir, crever, malheureusement) et repose sur la peur, à la fois du sacrifice du progrès (repartir, revenir), mais également des conséquences que font peser les dégradations environnementales sur la survie de la planète. Dans ce sens, la contrainte instituée (norme) est vue comme nécessaire. Le discours fait apparaître une volonté d'agir, de soi même, dans le sens d'une soumission bien comprise (accepter, respecter, préférer), voire consensuelle (charte, engagement). C'est par ce biais que s'établit la mise en relation des enjeux globaux avec les possibilités d'actions localisées de l'entreprise. Relation qui n'a rien d'univoque, les entreprises ayant sans doute intérêt à instaurer des normes qu'elles maîtrisent et qui, si elles se généralisent, leur confèrerait un avantage compétitif. Pour autant, le vocabulaire catastrophiste associé aux menaces systémiques est contrebalancé par l'emploi de termes porteur d'espoir et associés aux activités d'aide au développement (aider, changer, vivre), dans une mise en cohérence avec les activités économiques de l'entreprise (cahier, crèche, éducation, papier) ${ }^{10}$. Cette recherche de sens se fait sur un double

10 On notera ainsi que les activités d'aide au développement d'Ok, principalement tournées vers l'aide à l'enfance et vers l'éducation correspondent à la clientèle ciblée dans ses activités marchandes (le prêt-àporter pour enfants). 
mode marchand et industriel, car reposant sur une analogie clientéliste dont la cible marchande ou d'aide, est fondamentalement identique (l'enfant, chez Ok). Cette mise en cohérence repose également sur l'idée d'une action plus efficace qui porte sur un sujet qu'on connaît déjà à travers l'activité de l'entreprise (étude de marché, des modes, connaissance empirique du terrain, etc.). Les normes civiques de solidarités, avancées dans la critique qu'adresse le DD au système capitaliste, sont réinstaurées dans un ordre de valeur marchand: le nécessiteux (ou le sous-développé) reste appréhendé de manière clientéliste, ses besoins étant traités sur le mode de l'utilité.

\subsubsection{Discours associatif : la stratégie de maîtrise des normes.}

Alors que le discours entreprenarial fait assez peu référence aux dispositifs institués de contrainte, ceux-ci constituent, au contraire, un élément central des deux autres types de discours. Du côté associatif, les dispositifs légaux (de type réglementaires) sont relativement plus absents que le vocabulaire propre à la problématique des normes, dans le sens où elles relèvent de leur compétence. Celles-ci sont plus techniques lorsqu'elles portent sur la qualité de la production (norme industrielle, norme ISO, labellisation, etc.), ou plus diffuses lorsqu'elles portent sur des bonnes pratiques. Il s'agit alors d'encadrer le comportement des entreprises en définissant des objectifs considérés comme souhaitables au regard de la RSE (bonnes pratiques, guides, référentiels), et également à travers un système d'évaluation (mesurer, reporting) censé sanctionner les mauvais comportements au regard de l'opinion publique. Cette sanction, issue du monde commun de la renommée ou de l'opinion (Boltanski et Thévenot, 1991), s'avère être un important moteur de dynamique de la RSE dans la mesure où l'activité de l'entreprise doit être reconnue comme socialement légitime pour que ses produits rencontrent une demande effective (Capron, 2003 ; Champion et Gendron, 2005 ; Godard, 1993). La question de la maîtrise de la norme, notamment dans les critères d'évaluation ou de notation, est donc une question stratégique que les entreprises se doivent de maîtriser (par la création d'associations d'entreprises ou en participant aux comités de direction d'agences de notations, par exemple). Par ailleurs le rapport marchand, qui s'établit entre l'entreprise et l'agence qui fournit la prestation de notation sociale, représente une autre manière d'aborder ce problème, en même temps qu'il invite à réinterroger l'éthique de la firme (Capron, 2000). Dans cette perspective, l'ordre concurrentiel marchand fait peser de réels soupçons sur l'indépendance entre entreprise et agence. 


\subsubsection{Discours syndical : l'accent sur les parties prenantes.}

Quant au discours syndical, il est largement tourné vers le caractère légal (accord cadre, contrat, règle, loi, convention), en mettant l'accent sur la contrainte légitime gouvernementale, quelle que soit l'échelle d'exercice du pouvoir. À ce niveau, l'accent est mis sur le rôle actif des syndicats dans les négociations (signataire, signature, signé par), de même que sur leur place centrale dans ce processus. En effet, le discours donne à voir, à la différence des deux autres types, un tableau plus large des parties prenantes de l'entreprise (sous-traitant, salariés, comité, direction, administrateurs) tandis que le discours de l'action pose le syndicat comme médiateur volontaire (essayer), porté vers un consensus (fédératif, négocier, rassembler) entre parties aux objectifs parfois contradictoires (rapport de force, débat, exigent, revendicatif). En ce sens, l'acteur syndical est le seul dans le cas présenté ici, à reconnaître le rôle social structurant de l'entreprise à travers un traitement différencié des parties prenantes (rapport de force) (Gendron, 2004). La mise en cohérence du global au local intervient également dans ce type de discours, au niveau de l'articulation des conceptions (modèle théorique, réflexion, ligne, orientation) avec les enjeux pratiques de l'entreprise (expérience, terrain, besoin, situation, mise en ouvre concrète). L'organisation syndicale se présente, sous cet angle, comme un acteur privilégié de la RSE. Ses modalités d'action restent cependant dans des schémas de fonctionnement habituels (Stéphany, 2003) que cette dynamique ne permet pas, pour le moment, de renouveler. Notons que ce discours ne fait aucune référence aux thématiques relatives aux enjeux environnementaux, ou plus largement à ceux mis en jeu dans la problématique du DD, hormis quelques références temporelles qui indiquent une certaine projection vers l'avenir (futur, nouvel, devenir).

\subsection{Une typologie des entreprises.}

Deux critères permettent donc d'établir une typologie des acteurs : selon qu'ils mentionnent explicitement une référence à l'organisation globale du système (qu'elle soit vue à travers un mode marchand, civique ou en termes de parties prenantes) ; et selon la manière dont ils articulent les activités économiques aux enjeux globaux, c'est-à-dire la manière dont ils se positionnent par rapport aux normes de RSE ou de DD. On déduit le tableau 1 suivant des schémas 1 et 2 : 
Tableau 1 - Typologie des entreprises

\begin{tabular}{|c|c|c|c|c|}
\hline & & \multicolumn{3}{|c|}{ Position par rapport aux normes de RSE } \\
\hline & & Subie/ Eloignée & Les deux & $\begin{array}{l}\text { En prise directe/ } \\
\text { Mise en place }\end{array}$ \\
\hline \multirow{3}{*}{$\begin{array}{l}\text { Registre de } \\
\text { la globalité }\end{array}$} & $\begin{array}{l}\text { Référence } \\
\text { systémique }\end{array}$ & Ok & & $\mathrm{C}$ \\
\hline & $\begin{array}{l}\text { Niveau } \\
\text { intermédiaire } \\
\text { de référence }\end{array}$ & No & & \\
\hline & $\begin{array}{l}\text { Pas de } \\
\text { référence à } \\
\text { l'environneme } \\
\text { nt élargi de } \\
\text { l'entreprise }\end{array}$ & Bo & $\mathrm{EP}$ & $\mathrm{Mt}, \mathrm{Ca}, \mathrm{CE}, \mathrm{AL}$ \\
\hline
\end{tabular}

Cette mise en ordre typologique permet de faire apparaitre un critère fondamental (la norme) et un critère accessoire (la globalité). Finalement, la référence à une régulation du système économique, vue comme une nécessité, s'envisage dans les discours, via une certaine forme de normalisation qui établit un pont entre ce qui se passe dans, et hors de l'entreprise. Elle peut se faire sur le plan de la légalité (discours de $\mathrm{C}$ ), sur celui d'une acceptation des contraintes venues de l'extérieur (Ok, No), ou lorsque l'acteur considéré tente de produire ses propres normes, pour lui, ou pour les entreprises (par exemple par les associations). Au sein de cette catégorie, nous devons donc distinguer deux types d'acteurs: d'abord ceux qui édictent ces règlent, soit dans une perspective marchande de conservation d'un avantage compétitif (Ca), soit civique avec l'idée de garantir l'égalité des entreprises devant les dispositifs réglementaires (CE, AL), soit industrielle, de type ISO ou labellisation (EP) ; et ensuite ceux qui subissent ces normes en ce qu'elles les touchent au plus près de leur activité (Mt). Dans ce sens, EP joue un rôle particulier. En tant que groupement de grandes entreprises, cette association dispose du privilège de pouvoir choisir les règles que ses membres appliqueront. Il s'agit de maitriser les contraintes extérieures, notamment environnementales, qui pèsent sur la production (risque, conséquence, impact, déchet, coût, difficulté, assurance, sécurité). Ces contraintes sont mises en lien direct avec la fonction de l'entreprise (industrie, économie, offre, activité). Il s'agit donc d'un enjeu industriel hautement stratégique (Aggeri, 2004 ; 
Gendron, 2002) et reconnu comme tel (plan d'action, enjeu), puisqu'il s'agit de rester compétitif malgré la contrainte, en s'adaptant plus rapidement que les autres aux évolutions des règles mises en place (Godard, 1993). D'autres entreprises entretiennent un rapport marchand (commercial) ou industriel (exploitation, mise en valeur) avec la RSE (éventuellement via des activités d'aménagement de la nature) comme $\mathrm{Ca}$ ou Mt.

\section{Vers une typologie des enjeux de légitimité de RSE}

Chaque type d'entreprise entretient donc un lien différent avec les normes de DD. Celles-ci définissent autant de manières de se représenter les interactions de l'entreprise avec son environnement, c'est-à-dire de justifier l'étendue de sa responsabilité. Plusieurs modèles de légitimité peuvent être alors dégagés, définissant de nouveaux mondes communs. Ceux-ci se constituent ainsi en compromis entre grandeurs morales, mettant en jeu des éléments propres aux mondes communs identifiés par Boltanski et Thévenot. Il peut alors être intéressant de situer l'ensemble de ces modèles de légitimité qui émergent des discours de RSE, dans un contexte plus large de DD. Nous avons ainsi lancé une seconde analyse Alceste, à partir d'un corpus augmenté d'entretiens réalisés auprès d'élus et de personnels techniques, dans une vingtaine de collectivités territoriales (communes, intercommunalités, départements, régions) ${ }^{11}$. Sur cette dernière partie, un codage particulier a été réalisé permettant d'identifier trois types d'acteurs (les élus, les agents territoriaux, les entreprises). Au final, les discours analysés comportent des entretiens sur les politiques de DD des collectivités et de RSE des entreprises. Cette comparaison permet également de dessiner le positionnement relatif de deux parties prenantes importantes du DD local: les entreprises et les collectivités territoriales.

\subsection{La formalisation des enjeux de légitimité.}

Mais pour l'instant, on peut distinguer trois modèles d'appropriation du phénomène de DD par les entreprises. Ceux-ci sont largement dépendants du type d'activité qu'elles pratiquent et donc de la nature des biens ou des services produits. Lorsque l'activité est relativement déconnectée des enjeux de DD, les risques environnementaux ou de régulation sont perçus comme éloignés. Le discours alarmiste qui prévaut est alors extérieur à l'entreprise et il

11 De manière à neutraliser les effets de contexte, notamment liés au territoire, nous avons recueilli les discours des collectivités en charge des territoires sur lesquels sont implantés les acteurs de la RSE interrogés.

Economie et Institutions $-\mathrm{n}^{\circ} 15-2^{\mathrm{e}}$ semestre 2010 
est mobilisé au regard de grands principes humanistes et solidaires. L'entreprise privilégie alors des actions de type mécénat, aide au développement, etc. L'environnement ne joue pas de rôle de contrainte en prise directe avec les processus de production, mais est donc vu comme une opportunité de développement (Godard, 1993) permettant de réaliser des économies (d'énergie, recycler, récupérer) et d'améliorer l'image de l'entreprise (mondes industriel et du renom). Dans le cas de No et d'Ok, normalisation et enjeux de légitimité portent essentiellement sur les processus de production (égalité entre les hommes et les femmes, interdiction du travail des enfants, etc.). L'efficacité productive et la capacité à produire ainsi qu'à vendre à faible coût restent donc des modalités de légitimité largement partagées. Toutefois le recours à un vocabulaire patronal autocentré mettant en jeu des éléments domestiques (proximité, ancrage local) peut faire craindre une dérive néo-paternaliste (Stéphany, 2003) dans un compromis domestique/industriel. De même, l'utilisation d'un champ lexical issu du monde inspiré (imaginer, créer, métier, collaborateurs, projet), lorsqu'il est compromis avec des justifications marchandes peut induire une récupération capitaliste du phénomène (Boltanski et Chiapello, 1999), fatale pour les stratégies de DD dans l'entreprise ${ }^{12}$. La valorisation de l'image de l'entreprise (monde de l'opinion) est portée par des actions légitimes au regard d'un principe civique de solidarité qui reste déconnecté des enjeux industriels et marchands de l'entreprise (mécénat, aide au développement, etc.).

Lorsque l'activité de l'entreprise est relativement dépendante des enjeux de DD (comme c'est le cas par exemple de l'agroalimentaire avec l'environnement), mais que le produit fini en reste relativement éloigné (surgelé, conserve), il existe un risque mesuré pour l'entreprise, dans le sens où les processus de production ont des conséquences sur les consommateurs (notamment en termes de santé publique). L'environnement est alors perçu comme un coût, une difficulté à contourner, à adapter aux enjeux de responsabilité, pour ne pas détériorer l'image de l'entreprise. Dans ce cas, les enjeux de légitimité portent sur les processus de production, mais aussi sur la qualité des produits finis dont ils dépendent largement. La recherche d'efficacité se fait donc en tension avec des normes industrielles de respect de l'environnement ou de qualité des produits. Ces normes sont acceptées par l'entreprise dans la mesure où elles répondent à un principe civique de non discrimination concurrentielle, en cohérence avec un principe marchand. On

12 Par ailleurs, Gendron (2000) montre que la capacité du capitalisme à récupérer les critiques qui lui sont adressées, trouve son corollaire théorique dans la possibilité de réduire les trois représentations dominantes du phénomène de RSE aux postulats économiques néoclassiques de base. 
s'évertue par exemple chez Bo à respecter la législation anti-OGM, alors que cette technologie est vue en interne comme un progrès. Dans cette optique, les questions de l'acceptation sociale et de légitimité du produit au regard de l'opinion publique, restent déterminantes, comme l'illustre l'exemple de Perrier cité par Godard (1993) $^{13}$. Il existe alors un risque marchand qui reste relativement faible en France (Laville, 2002) de défection consumériste (boycott).

Dans le dernier cas, il existe une opportunité marchande liée aux enjeux de RSE ou aux risques environnementaux, dans la mesure où elle constitue l'activité principale de l'entreprise ou de la branche, dans une stratégie de différenciation des services ou des produits (Jolly, 2006). Dans le cas de Mt, la fourniture de revêtements routiers "durables" se différencie d'un simple aménagement de la nature. Chez $\mathrm{Ca}$, on diversifie les activités de l'entreprise par le conseil stratégique en DD. Il s'agit alors de mettre en place des investissements, parfois coûteux, de manière rendre visible la différence effective des produits ou des services. Dans le dernier cas, la dynamique de RSE ou la protection de l'environnement est instrumentalisée comme élément de différenciation. En ce sens, l'enjeu de légitimité porte sur le produit (ou le service) final. La production et l'échange restent portés par des modalités propres au monde marchand dont les principes sont contraires à ceux de la RSE, dans le mesure où ils ne relèvent en rien d'une "nouvelle éthique " de l'entreprise (Gendron, 2000). Il existe donc deux dérives possibles: l'une marchande puisque le DD est subordonné au critère d'utilité; et une dérive techniciste (industrielle), dans la mesure où la logique concurrentielle conduit les entreprises à s'appuyer sur des moyens de diagnostic (propres au monde industriel) toujours plus poussés, qui éloignent toujours plus les objectifs fixés et risquent de décourager la demande en conseil de stratégie de DD (Stéphany, 2003).

13 En 1990, le groupe Perrier avait décidé de retirer la totalité des stocks de bouteilles des circuits de distribution américains alors qu'un élément non naturel était présent dans certains lots seulement. L'ampleur de cette mesure visait à restaurer la confiance du consommateur en ôtant tout doute sur la qualité des produits mis en vente. 
Tableau 2 - Enjeux de légitimité

\begin{tabular}{|c|c|c|c|}
\hline & No, Ok & Bo & $\mathrm{Ca}, \mathrm{Mt}$ \\
\hline $\begin{array}{l}\text { Rapport à } \\
\text { l'environnement } \\
\text { (ou à la } \\
\text { RSE)/Risque }\end{array}$ & $\begin{array}{l}\text { Activité éloignée des } \\
\text { problématiques } \\
\text { environnementales } \\
\text { Le discours du risque } \\
\text { n'est pas mobilisé. } \\
\text { Catastrophisme } \\
\text { extérieur à l'entreprise }\end{array}$ & $\begin{array}{l}\text { Activités en lien avec les } \\
\text { enjeux } \\
\text { environnementaux. } \\
\text { Il existe un risque pour } \\
\text { l'entreprise, lié à des } \\
\text { enjeux de santé } \\
\text { publique et qui mettent } \\
\text { en jeu les processus de } \\
\text { production }\end{array}$ & $\begin{array}{l}\text { Activités directement } \\
\text { liées à l'environnement } \\
\text { ou à la RSE. } \\
\text { Il existe une opportunité } \\
\text { marchande en rapport } \\
\text { avec les risques } \\
\text { environnementaux. }\end{array}$ \\
\hline $\begin{array}{l}\text { Perception de } \\
\text { l'environnement }\end{array}$ & $\begin{array}{l}\text { La contrainte (ou la } \\
\text { norme) } \\
\text { environnementale est } \\
\text { vue comme une } \\
\text { opportunité. } \\
\text { - Réalisation } \\
\text { d'économies. } \\
\text { - Amélioration de } \\
\text { l'image. }\end{array}$ & $\begin{array}{l}\text { L'environnement est vu } \\
\text { comme un coût, une } \\
\text { difficulté. }\end{array}$ & $\begin{array}{l}\text { L'environnement est vu } \\
\text { comme un coût } \\
\text { (investissement) et une } \\
\text { opportunité de } \\
\text { développer un nouveau } \\
\text { marché ou une nouvelle } \\
\text { branche d'activité. }\end{array}$ \\
\hline $\begin{array}{l}\text { Enjeu de } \\
\text { légitimité }\end{array}$ & $\begin{array}{l}\text { Uniquement sur les } \\
\text { processus de production }\end{array}$ & $\begin{array}{l}\text { Sur les processus, mais } \\
\text { également sur la qualité } \\
\text { des produits finis. }\end{array}$ & $\begin{array}{l}\text { Sur les produits finis ou } \\
\text { les services. }\end{array}$ \\
\hline $\begin{array}{l}\text { Monde légitime } \\
\text { en tension avec } \\
\text { l'opinion } \\
\text { publique et le } \\
\text { marché }\end{array}$ & $\begin{array}{l}\text { Tension entre les } \\
\text { mondes industriels et } \\
\text { inspirés et entre les } \\
\text { mondes industriel et } \\
\text { domestique. } \\
\text { - Risque de dérive néo- } \\
\text { paternaliste. } \\
\text { - Risque de dérive néo- } \\
\text { libérale. }\end{array}$ & $\begin{array}{l}\text { Tension avec les mondes } \\
\text { civique et industriel. } \\
\text { - Risque de dérive } \\
\text { consumériste sur } \\
\text { l'efficacité de } \\
\text { l'entreprise. }\end{array}$ & $\begin{array}{l}\text { Tension avec le monde } \\
\text { marchand et industriel. } \\
\text { - Risque de dérive } \\
\text { marchande. } \\
\text { - Risque de dérive } \\
\text { techniciste. }\end{array}$ \\
\hline
\end{tabular}

\subsection{Une mise à distance des enjeux de développement durable}

Ce tableau fait apparaître un espace relativement peu stabilisé de légitimité. La proximité des activités de l'entreprise avec les enjeux de DD, notamment environnementaux, apparaît comme un facteur 
majeur de cette disparité. Celle-ci contribue à mettre à distance le DD. De fait, l'application des principes de DD dans l'entreprise, reste largement conditionnée à la possibilité qu'elles se ménagent, de les maîtriser.

En résumé, le DD comme stratégie d'entreprise se différencie des principes édictés par les instances internationales. Il nous reste à montrer que l'écart entre principes et mise en application ne résulte pas d'une simple difficulté opératoire, d'un "fossé d'implémentation ", en nous appuyant sur une étude comparée des discours de DD dans les entreprises et dans les collectivités territoriales. Les résultats fournis par l'analyse sont résumés dans le schéma 3, qui fait apparaître quatre classes de discours spécifiques. On constate, sans surprise, l'apparition d'une classe, la dernière, particulière aux entreprises - c'est d'ailleurs le terme le plus significatif ce qui semble valider l'hypothèse d'un repli sur ses enjeux et ses fonctions.

Schéma 4 - Les classes de discours du DD.

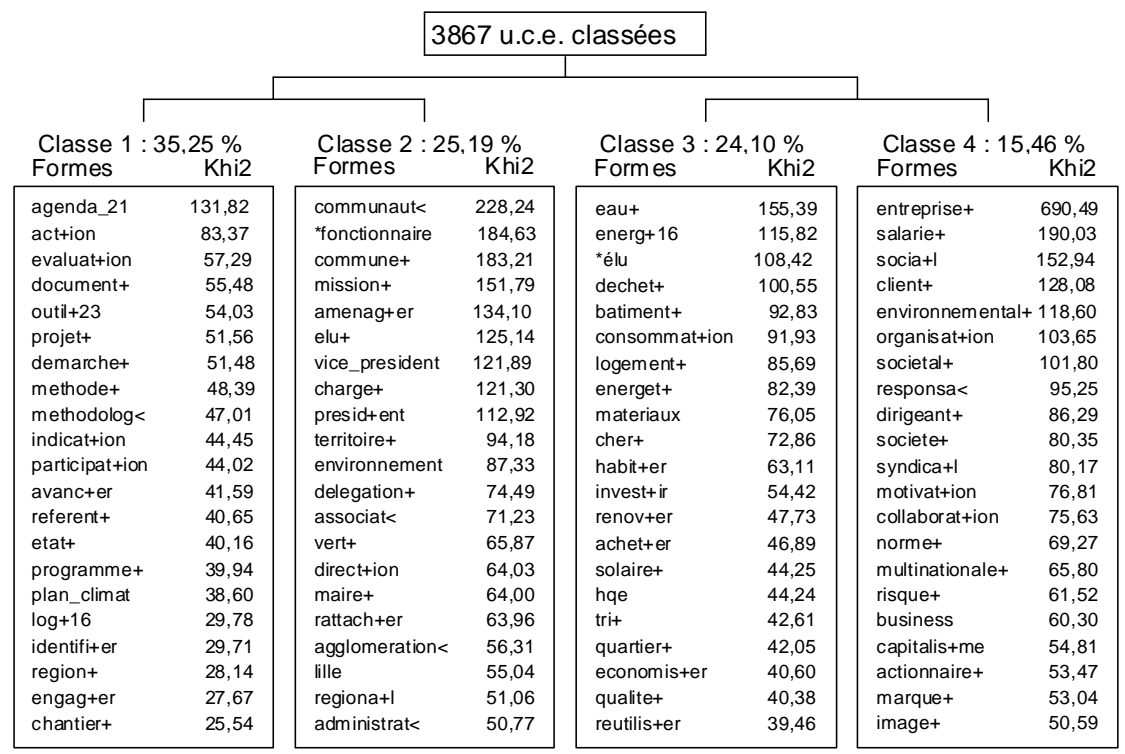

Les trois autres classes sont spécifiques au discours des collectivités :

La classe 1 est constituée d'une classe de vocabulaire propre aux outils, dans une tentative de définition d'une méthodologie de développement durable (évaluation, participation, échange). Les marqueurs d'actions (verbes) propres à cette classe 
semblent indiquer que les outils de $\mathrm{DD}$, particulièrement le projet d'agenda 21, constituent une passerelle entre le travail des fonctionnaires (animer, engager, proposer, questionner) et celui des élus (voter, adopter, piloter). Cette collaboration se fait dans la gestion des enjeux concrets du présent (tout de suite, en cours, régulier).

La classe 2 est représentative du discours du personnel technique. Ces derniers mobilisent un vocabulaire tourné vers l'organisation administrative des collectivités $\left(\right.$ dgs $^{14}$, direction, structure, service, hiérarchie, organigramme, secrétaire) qui marque une division des compétences cloisonnées, mais également un partage des responsabilités entre élus (président, vice président, maire) et techniciens du DD. Dans ce type de discours, l'accent est particulièrement mis sur la dimension environnementale du DD comme modalité renouvelée d'aménagement du territoire (rural, urbain, bassin minier, espace), notamment dans l'articulation des différentes échelles (communauté urbaine, commune, agglomération, département, régional, pays).

La classe 3 représente des enjeux de politiques publiques et est mobilisée dans les discours d'élus. Ces enjeux peuvent être traditionnels (logement, bâtiment, consommation,), ou renouvelés par la problématique du DD (eau, énergie solaire, récupérer, trier, qualité, déchets, friche).

Les collectivités sont aussi confrontées à des enjeux de légitimité comparables à ceux des entreprises. Pour produire pleinement ses effets, une politique publique doit, en effet, remporter l'adhésion du publique ciblé. La qualité des services publics dépend aussi de l'engagement des usagers (Ostrom, 1998). Dans la mise en application du DD via les agendas 21 , on retrouve donc un vocable relatif au monde du renom, similaire à celui de l'entreprise (image). Il s'agit en effet d'une démarche à valoriser au moyen d'outils de communication (publication, document). Cependant les mondes communs en compromis qui semblent s'esquisser au niveau des collectivités, mettent en jeu des éléments du monde civique plutôt que marchand ou industriel. La recherche de légitimité passe par un renouvellement des modalités démocratiques. Traditionnellement la légitimité des politiques publiques vient des mandats acquis par les élus au cours d'une épreuve électorale. Avec le DD, l'adhésion à la démarche en interne ou en externe dans les politiques publiques, est requise dans une épreuve de participation ou de concertation autour du projet (échange, partage, partenaire, rencontre).

14 "Directeur général des services " ou " direction générale des services ". 


\subsection{Entreprises et collectivités, un lien univoque.}

Les pratiques de DD des organisations dessinent finalement un ensemble d'enjeux de légitimité peu stabilisé. Les normes - de DD ou de RSE, apparaissent alors comme relativement peu contraignantes. C'est donc, à l'inverse, dans la manière dont les organisations légitiment leurs actions (productives ou politiques) qu'elles définissent leur conception du DD. Ainsi, pour les entreprises, la RSE constitue un moyen de valoriser leur image en répondant à une certaine demande sociale en matière de régulations du capitalisme. Pour les collectivités territoriales, il s'agit de changer de paradigme administratif en remettant en cause la sectorisation des divers volets de politiques, en essayant de porter une démarche transversale aux services et en s'appuyant sur des procédures participatives ou de concertation. Cette tension entre les manières de légitimer les produits de l'entreprise ou de la collectivité territoriale, induit une fracture notionnelle qui met à distance la conception publique consensuelle du DD, dans les entreprises. Ce phénomène est également visible dans l'asymétrie des relations qu'entretiennent ces deux acteurs, en matière de gouvernance territoriale du DD. En effet, alors que les collectivités voient les entreprises comme des acteurs-clés du territoire, ces dernières considèrent les collectivités comme des organismes de régulation qui accroissent la pression normative et instituent de nouvelles contraintes.

Dans la classe sémantique propre aux élus (classe 3 ) on mobilise pourtant un vocable proche de celui de l'entreprise, dans laquelle la collectivité se décrit comme une organisation fournissant un service aux habitants, en réponse direct à leurs besoins: déplacements (autoroute, bagnole, voiture, véhicule, vélo, train), logement (bâtiment, quartier), traitement des déchets (tri, poubelle), fourniture d'énergies (eau, gaz), de services publics (école), etc. Les préoccupations sont assez largement tournées vers l'économie (prix, produit, ressource, offre), dans un contexte problématique (coût, effort, obligation, cher). Les marqueurs d'action (verbes) font également référence aux fonctions productives et industrielles des collectivités (acheter, consommer, économiser, investir, payer, utiliser). La différence avec le vocable d'entreprise (classe 4) réside essentiellement dans l'utilisation d'un discours d'action tourné vers les pratiques internes à l'organisation en matière environnementale (arroser, protéger, récupérer, recycler, réduire, différencier et raisonner). À cet égard, la nature est perçue comme un Bien en soi qu'il s'agit de préserver en rapport à un principe de survie (Capron, 2000 ; Harribey, 1998) et non dans une logique industrielle de préservation des capacités productives des ressources. L'action se dirige également hors de la collectivité qui tente d'agir sur le 
comportement d'autres acteurs (allumer, dégrader, jeter) dans le sens souhaité (isoler, rapporter (des objets usagés), recycler, réduire). Dans cette optique, le marqueur de la personne (pronom personnel) ils apparait comme le plus significatif de cette classe.

Par ailleurs, les nombreux marqueurs de temps et d'espace induisent une préoccupation d'équilibrage des enjeux du présent et du futur, du global et du local, en cohérence avec le principe de solidarité du DD (génération future, actuellement, avenir, déjà, demain, maintenant, tard, ici, ailleurs). Cela contraste avec les marqueurs temporels mobilisés dans les discours d'entreprises où seul le terme aujourd'hui apparaît comme significatif ${ }^{15}$. Les références temporelles mobilisées par les entreprises semblent se focaliser sur les problèmes qu'elles rencontrent dans le présent, mettant éventuellement en œuvre une démarche pragmatique de court terme.

Quoi qu'il en soit, le lien entreprises/collectivités est donc marqué par une certaine asymétrie. Les organisations publiques semblent prendre en compte les dimensions internes de leur activité en essayant d'avoir une influence sur le comportement d'autres acteurs. De ce point de vue, elles tentent de mettre en place des moyens marchands de coopération, dans une tentative de création de nouvelles filières, de nouveaux métiers, via les commandes publiques (instauration de clauses dans la passation des marchés publics). Un levier d'action relativement efficace sur le comportement des entreprises réside également dans l'écoconditionnalité des financements et subsides accordés aux entreprises, ou dans l'introduction de clauses éthiques dans la passation des marchés publics. À l'opposé, les entreprises mobilisent un vocable d'action largement tourné vers la contrainte (imposer, respecter, modifier, négocier, réglementer) en opposition avec ses fonctions (créer, vendre) et divers éléments de son entourage matériel (marque, magasin, produit, usine, industrie). Finalement, la mise à distance s'effectue par le recours particulièrement significatif de démonstratifs (celui, celle), éventuellement dans une chosification (ceci, cela, c'est, ce), pour désigner ce qui n'est pas dans l'entreprise : l'autre.

\section{Conclusion}

Les analyses comparées des discours de DD des entreprises et des collectivités territoriales, montre donc que les premières, bien qu'elles revendiquent une responsabilité étendue, restent assez largement dominées par les exigences de profitabilité. Dans cette

15 Rappelons que dans l'analyse précédente, le terme "futur " est significatif du discours syndical sans qu'aucun marquer temps identifié dans l'analyse n'apparaisse. 
optique, la RSE apparaît comme une nouvelle modalité de légitimité inhérente à la production de biens ou de services qui doit être analysée au regard de la nature des biens ou services produits. Elle se cristallise dans une certaine vision du DD, dans une traduction qui met l'accent sur les rapports de l'entreprise avec l'ensemble de la société (Gendron, 2000), y compris la protection de l'environnement, mais laisse de côté les questions de solidarité inter ou intragénérationnelle (Aggeri, 2004).

Selon nous certains éléments plaident cependant en faveur d'une démarche assumée, une troisième voie entreprenariale, entre RSE et recherche du profit à tout prix. Cette troisième voie pourrait passer par la mise en application d'une démarche de production transversale appliquée à l'entreprise. Dans les collectivités territoriales, en effet, la transversalité est vue comme une condition incontournable de la mise en application d'une politique de DD. Or la division du travail entre élus et personnels techniques, mais également entre les services auxquels échoit l'implémentation des politiques sectorielles, continue de dominer largement la réalité des organisations territoriales (Emelianoff, 2005). Dans les entreprises, les modifications managériales de gestion qui réorganisent les techniques de production, aboutissent à une remise en cause de l'organisation hiérarchique du travail (Boltanski et Chiapello, 1999). On passe alors d'un schéma productif vertical à un schéma en réseau (Stéphany, 2003). Ainsi les parties prenantes à l'activité de l'entreprise ont un rôle à jouer, ce que met en évidence le vocabulaire de la forme entreprise (client, syndical, sous-traitant, actionnaire, dirigeant, banque, salarié). Il semble donc, de ce point de vue, que l'entreprise ait déjà réalisé sa mutation organisationnelle propice à l'instauration d'une stratégie transversale de DD. Reste, dans ce réseau des parties prenantes, à déterminer les formes légitimes d'attribution des responsabilités relatives, pour que chacun puise jouer son rôle au regard des enjeux sociétaux de DD.

\section{Bibiliographie}

Aggeri F., (2004), "Les entreprises françaises et le développement durable", Regards sur l'actualité, (302), pp. 57-65.

Aggeri F. et O. Godard, (2006), "Les entreprises et le développement durable", Entreprises et histoire, 45 (4), pp. 6-19.

Boidin B. et al., (2007), Responsabilité sociale et environnementale de l'entreprise : interaction et logiques d'acteurs dans la construction de normes, Villeneuve d'Ascq, Rapport de recherche CLERSE-IFRESI, 231.

Boltanski L. et E. Chiapello, (1999), Le nouvel esprit du capitalisme, Paris, Gallimard. 
Boltanski L. et L. Thévenot, (1991), De la justification. Les économies de la grandeur, Paris, Gallimard.

Brundtland G. H., (1987), Notre avenir à tous, Montréal (Édition 1988), Les Éditions du Fleuve.

Capron M., (2000), "Vous avez dit "éthique" ?" Revue du MAUSS, 15 (1er semestre), pp. 271-277.

Capron M., (2003), "Pourquoi et comment évaluer le comportement des entreprises en matière de développement durable ?" dans Najim A. et al. (dir.), Les entreprises face aux enjeux du développement durable, Karthala, pp. 111-131.

Champion E. et C. Gendron, (2005), "De la responsabilité sociale à la citoyenneté corporative : l'entreprise privée et sa nécessaire quête de légitimité", Nouvelles pratiques sociales, 18 (1), pp. 90-103.

Coutrot T., (1998), L'entreprise néo-libérale, nouvelle utopie capitaliste? Enquête sur les modes d'organisation du travail, Paris, La Découverte.

Emelianoff C., (2005), "La ville durable en quête de transversalité", dans Mathieu N. et Y. Guermond (dir.), La ville durable, du politique au scientifique, Paris, Éd. Cemagref, INRA, pp. 129-142.

Gendron C., (2000), "La responsabilité sociale", Revue du MAUSS, 15 (1er semestre), pp. 320-325.

Gendron C., (2002), "De la reconnaissance à l'engagement : représentation et éthique de l'environnement et du développement durable chez les hauts dirigeants du Québec", Entreprise Éthique, (16), pp. 41-47.

Gendron C., (2004), "Entre responsabilité sociale et entreprise, une éthique d'intérêts ?" Entreprise Éthique, (21), pp. 100-104.

Godard O., (1990), "Environnement, mode de coordination et systèmes de légitimité : analyse de la catégorie de patrimoine naturel", Revue économique, (2), pp. 215-242.

Godard O., (1993), "Stratégies industrielles et convention d'environnement : de l'univers stabilisé aux univers controversés", INSEE Méthodes, (39-40), pp. 145-174.

Godard O., (1994), "Le développement durable : paysage intellectuel", Nature, Sciences, Sociétés, 2 (4), pp. 309-322.

Godard O., (2004), "De la pluralité des ordres. Les problèmes d'environnement et de développement durable à la lumière des théories de la justification", Géographie, Économie, Société, (6), pp. 303-330.

Harribey J.-M., (1998), Le développement soutenable, Paris, Economica.

IUCN, (1980), World Conservation Strategy: Living Resource for Sustainable Development, International Union for Conservation of Nature and Natural Resources (IUCN), United Nations Environment Program (UNEP) and World Wildlife Fund (WWF), 
http://www.nembro.net/agenda21locale/doc/1980-

The $\% 20$ world $\% 20$ conservation $\% 20$ strategy.pdf.

Jolly C., (2006), L'entreprise responsable : sociale, éthique, "verte"... et bénéficiaire?, Paris, Le Félin.

Laville E., (2002), L'entreprise verte : le développement durable change l'entreprise pour changer le monde, Editions Village Mondial.

Ostrom E., (1998), "The Comparative Study of Public Economies", American Economist, 42 http://www.indiana.edu/ workshop/publications/materials/seidma nweb.htm, pp. 3-17.

Stéphany D., (2003), Développement durable et performance d'entreprises, Paris, Editions Liaisons.

Thévenot L. et al., (2000), "Forms of Valuing Nature: Arguments and Modes of Justification in French and American Environmental Disputes", dans Lamont M. et L. Thévenot (eds.), Rethinking Comparative Cultural Sociology: Repertoires of Evaluation in France and the United States, Cambridge, Cambridge University Press, pp. 229-272.

UE, (2001), Livre Vert de la Commission européenne. Promouvoir un cadre européen pour la responsabilité sociale des entreprises, Bruxelles, Commission des Communautés européennes, http://eurlex.europa.eu/LexUriServ/site/fr/com/2001/com2001_0366fr01.pdf

Zaccaï E., (2002), Le développement durable : dynamique et constitution d'un projet, Bruxelles ; New York, P.I.E.-Peter Lang. 